\title{
The Verification of iPlan Commissioning by Radiochromic EBT2 Films
}

\author{
Maria F. Chan, Qinghui Zhang, Jingdong Li, Preeti Parhar, Karen Schupak, Chandra Burman \\ Department of Radiation Oncology, Memorial Sloan-Kettering Cancer Center, Basking Ridge, USA \\ Email: \{chanm, zhangq, lij2, parharp, schupakk, burmanc\}@mskcc.org \\ Received April 2, 2012; revised April 28, 2012; accepted May 7, 2012
}

\begin{abstract}
Purpose: To evaluate the measured dose distributions using radiochromic EBT2 films for small fields in iPlan (BrainLab) commissioning. Methods: Radiochromic EBT2 films were irradiated with 6 MV photons on a Varian Trilogy linac using polystyrene phantoms. The measurements included dose profiles and depth doses for field sizes of $1 \times 1,2 \times 2,3$ $\times 3,4 \times 4$, and $10 \times 10 \mathrm{~cm}^{2}$ etc. The dose profile measurements were taken at the depth of $5 \mathrm{~cm}$. The calibration films were irradiated at $\mathrm{d}_{\max }(1.4 \mathrm{~cm})$ for doses up to $6 \mathrm{~Gy}$. Films were scanned using an Epson 10,000 XL flatbed scanner with 72 dpi resolution. Pixel values were converted to doses using the established calibration-curve. The $2 \mathrm{D}$ dose distributions were generated from the film data analysis. In-house software was utilized to compare the measured doses from films with the treatment planning data. In addition, selected patients' SRS fields were also measured with the EBT2 films for comparison with iPlan. An EDGE ${ }^{\mathrm{TM}}$ detector was also used to check the central-axis doses for the SRS patients' measurements. Results and Discussion: The measured planar dose distributions achieved more than $98 \%$ and $95 \%$ passing rates with a set of $2 \% / 2 \mathrm{~mm}$ dose and DTA criteria for all square fields and all patient treatment fields $(<5$ $\left.\times 5 \mathrm{~cm}^{2}\right)$, respectively. Agreement with measurement data with the $\mathrm{EDGE}^{\mathrm{TM}}$ detector at the central axis $( \pm 1 \%)$ was found with the plan data. This is the first report for SRS small photon-field measurement using the latest radiochromic film, EBT2. The results shown in this work indicate that the use of EBT2 film provides accurate dosimetry measurements for small photon beams. The measurements show excellent agreement with the iPlan TP commissioning data. Conclusions: The patient-specific EBT2 film QA for iPlan SRS patients showed good results. The EBT2 films could potentially be a useful dosimeter in verification of commissioning as well as patient-specific QA for SRS cases.
\end{abstract}

Keywords: Radiochromic Film; Dosimetry; Radiosurgery; Commissioning; Radiotherapy

\section{Introduction}

Radiotherapy beams become smaller and conform more tightly to the tumor shape while radiation delivery equipment is more capable of delivering small fields. Stereotactic radiosurgery (SRS) has been delivering very small fields for many years either using GammeKnife or linacbased equipment. The shape of each beam from linacbased system can be controlled by collimators as small as a few $\mathrm{mm}$. The clinical dosimetry of small fields is challenging due to nonequilibrium conditions created as a consequence of the secondary electron track lengths and the source size projected through the collimating system [1-6]. Moreover, SRS requires high precision and accuracy in the calculation of dose distributions. One of the possible errors in SRS dosimetry is in the correct selection of the detector used since it requires high spatial resolution $[7,8]$.

Radiochromic EBT film has been established as an

*EBT2 film for iPlan commissioning. accurate quantitative 2D dosimeter with fine spatial resolution for applications in external beam and brachytherapy, including IMRT QA, commissioning of treatment modalities and verification of TPS [9-30]. The recently introduced radiochromic film, EBT2, a successor of EBT film, has dosimetric characteristics which are similar including its weak energy dependency [15,31-34]. The use of EBT2 film for IMRT QA and verification of calculated 2D dose distributions has also been reported [3537]. In our institution, iPlan is used for single-fraction SRS brain treatments. This work presents that we have verified the commissioning of iPlan with radiochromic EBT2 films.

\section{Materials and Methods}

\subsection{Treatment Planning Calculations}

A virtual phantom was used in the iPlan treatment planning system (TPS) (version 4.1, BrainLab, Germany) to generate depth doses and profiles for field sizes of $1 \times 1$, 
$2 \times 2,3 \times 3,4 \times 4$, and $10 \times 10 \mathrm{~cm}^{2}$. The central axis doses and field size factors were also generated using the TPS.

\subsection{Irradiation of EBT2 Films in Polystyrene Phantom}

Radiochromic EBT2 films from lot\# A09031001A (Ashland Inc., Wayne, NJ) were irradiated with $6 \mathrm{MV}$ photons on a Varian Trilogy linac using a polystyrene phantom $\left(25 \times 25 \times 15 \mathrm{~cm}^{3}\right)$. The measurements included dose profiles and depth doses for field sizes of $1 \times 1,2 \times$ $2,3 \times 3,4 \times 4$, and $10 \times 10 \mathrm{~cm}^{2}$. The field sizes were defined by multileaf-collimator (MLC) and the jaw sizes were set $1 \mathrm{~cm}$ outside the MLC edge. The depth doses were measured by positioning the films vertically parallel to the beam's CAX sandwiched by 2 slabs of polystyrene phantoms. The dose profile measurements were taken at the depth of $5 \mathrm{~cm}$. The calibration films were irradiated at $\mathrm{d}_{\max }(1.4 \mathrm{~cm})$ for doses up to $6 \mathrm{~Gy}$. Calibration film doses were standardized against the ion chamber (Standard Imaging Exradin A-12 $0.65 \mathrm{cc}$ thimble chamber with ADCL calibration) measurement at the same location and depth. The output of the Trilogy linac was calibrated per AAPM TG-51 protocol.

\subsection{Scanning of Films}

All measured EBT2 films, calibration, background films were scanned at the same central location and orientation on an Epson 10,000 XL flatbed scanner in transmission mode. There were at least 2 films for each measured set. The polymer emulsion coating direction of the film was parallel to the scanning direction on the scanner bed. The settings of 48 bit color and $72 \mathrm{dpi}(0.035 \mathrm{~cm}$ per pixel) were used, color correction was disabled, and files were saved in TIFF format. The calibration films, background films, and experimental films were scanned one at a time at least 16 hours after irradiation.

\subsection{Data Processing and Analysis}

The red channel data were extracted using a software, ImageJ v1.38 (National Institute of Health, Bethesda, $\mathrm{MD}$, http://rsb.info.nih.gov/ij/). The pixel values (PV) in the processed image file were converted to net optical density (NOD), defined by the following equation.

$$
\mathrm{NOD}=\log \left(\mathrm{PV}_{\text {background }} / \mathrm{PV}\right)
$$

For each calibration or background film, the average of the pixel values in an area of about $1 \times 1 \mathrm{~cm}^{2}$ at the film center was calculated and assigned as the PV of the film. $\mathrm{PV}_{\text {background }}$ is the $\mathrm{PV}$ of the background film.

The NOD values of the calibration films were plotted against dose values (in cGy) to form the calibration curve.
The calibration curve was fitted by a third order polynomial. Conversion from net optical density readings to doses was achieved based on the polynomial fit to the calibration curve.

The 2D dose distributions were generated from the film data analysis. In-house software was utilized to compare the measured doses from films with the corresponding treatment planning data. In addition, selected patients' SRS beams were measured with the EBT2 films for comparison with iPlan results. An $\mathrm{EDGE}^{\mathrm{TM}}$ detector (Sun Nuclear Corp) was also used to check the central-axis doses for the SRS treatment fields. The depth of measurement was also at $5 \mathrm{~cm}$. The $\mathrm{EDGE}^{\mathrm{TM}}$ detector is an ultra small dosimetry detector for small field measurement which width and length is $0.8 \mathrm{~mm}$ each.

\section{Results and Discussions}

\subsection{Calibration Curves}

The NOD values of the calibrated EBT films of lots\# A09031001A were plotted against dose values, as shown in Figure 1. A 3rd order polynomial was obtained for dose conversion from NOD values, as shown in Equation (2).

$$
\mathrm{D}(\mathrm{cGy})=\mathrm{c}_{0}+\mathrm{c}_{1} \mathrm{NOD}+\mathrm{c}_{2} \mathrm{NOD}^{2}+\mathrm{c}_{3} \mathrm{NOD}^{3}
$$

where the coefficients are $\mathrm{c}_{0}=0.29005, \mathrm{c}_{1}=671.03, \mathrm{c}_{2}=$ $1404.4, c_{3}=2214$.

\subsection{Percent Depth Doses along the Central Axis}

The central-axis percent depth dose (PDD) values were plotted against the depth in water, as shown in Figures 2(a), 2(b) and 3(a), 3(b). Figures 2(a) and 3(a) are the 2D isodoses plots along depths and Figures 2(b) and 3(b) are the 1-dimentional PDD curves for MLC settings of 1 $\times 1 \mathrm{~cm}$ and $2 \times 2 \mathrm{~cm}$, respectively. The solid lines in isodoses were from iPlan and dotted lines were from EBT2 film.

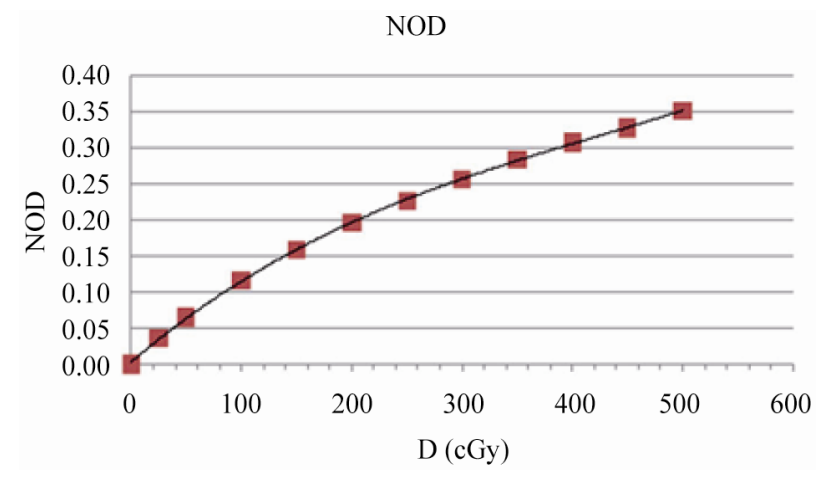

Figure 1. Calibration curve for EBT2 films (lot\# A09031001A). 


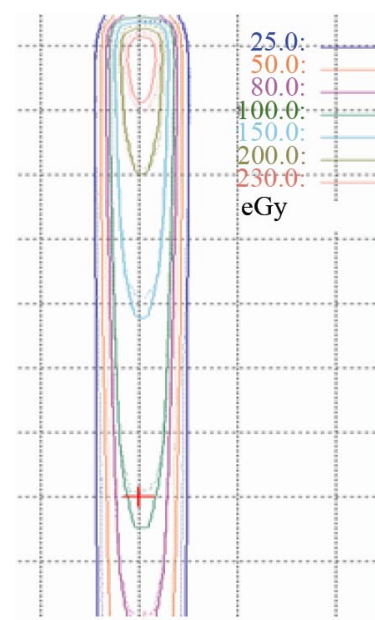

(a)



(b)
Figure 2. (a) $1 \times 1 \mathrm{~cm}$ MLC $(2 \times 2 \mathrm{~cm}$ jaw); (b) TP: pink, film: white.

\subsection{Beam Profiles and Isodoses Overlay}

Figures 4(a) and 5(a) were the isodoses overlay between EBT2 film data and iPlan planar doses at the depth of 5 $\mathrm{cm}$ (TP: solid lines, film: dotted lines). The values of percent doses and off-axis ratios were plotted against the lateral distance at $5 \mathrm{~cm}$ depth as shown in Figures 4(b), 4(c), 5(b), 5(c). Figures 4 and 5 were for the field sizes of $1 \times 1,2 \times 2 \mathrm{~cm}$, respectively. The passing rates of Gamma index $(2 \% / 2 \mathrm{~mm})$ for all MLC-defined (square) field sizes in this study were above $98 \%$.

\subsection{Patient-Specific QA}

In addition, patient-specific SRS QA has been performed to ensure the accuracy of the iPlan commissioning. The Gamma Index in this work was used $2 \% / 2 \mathrm{~mm}$ and the passing rates of all SRS fields were better than $95 \%$. Figures 6(a)-(c) and 7(a)-(c) show 2 of the 10 patient's SRS-field measurements data vs. those generated from iPlan. Solid lines are iPlan isodoses and dotted lines were EBT2 data.

The measured planar dose distributions achieved more than $95 \%$ passing rates with a set of $2 \% / 2 \mathrm{~mm}$ dose and DTA criteria for all patient treatment fields $\left(<5 \times 5 \mathrm{~cm}^{2}\right)$. The uncertainties of the measured doses were estimated following the method described in the EBT film studies [20,37]. Combining the Type A (random, statistical) and Type B (non-random, systematic) uncertainties, the uncertainties of the measured doses at individual pixels were about $4 \%$. Although we measured the field sizes up to $10 \times 10 \mathrm{~cm}^{2}$, we have only shown the small sizes PDD and profiles in the result section because small field is the main focus in this study. In addition, agreement with measurement data using the $\mathrm{EDGE}^{\mathrm{TM}}$ detector (Sun Nuclear Corp) at the central axis $( \pm 1 \%)$ was found with the plan data. The results shown in this work indicate that the use of EBT2 film provides accurate dosimetry measurements for small photon fields. The measurements show excellent agreement with the iPlan TP commissioning data. There have been reports of measuring depth doses and beam profiles using EBT films positioned vertically parallel to the beam's CAX in a water tank [21]. However, van Battum et al. [21] found dose discrepancy between EBT and ion chamber data in the superficial depths within $0.9 \mathrm{~cm}$. They attributed the discrepancy to possible error in the ion chamber data in this region.

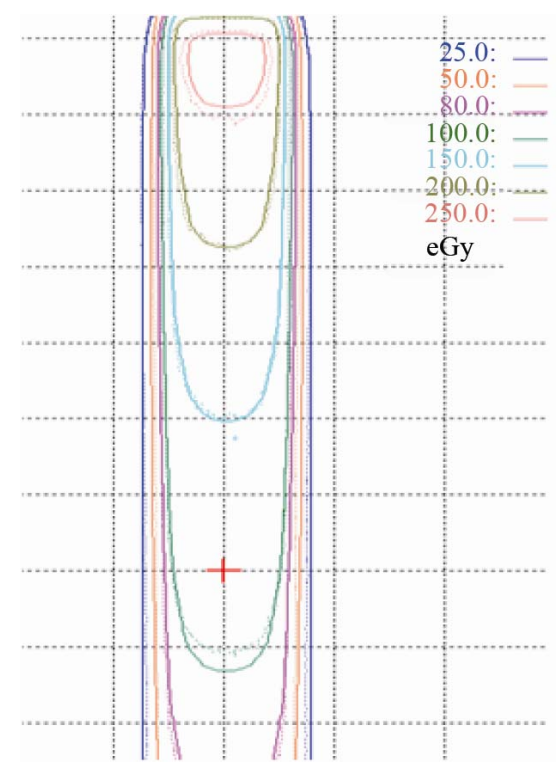

(a)

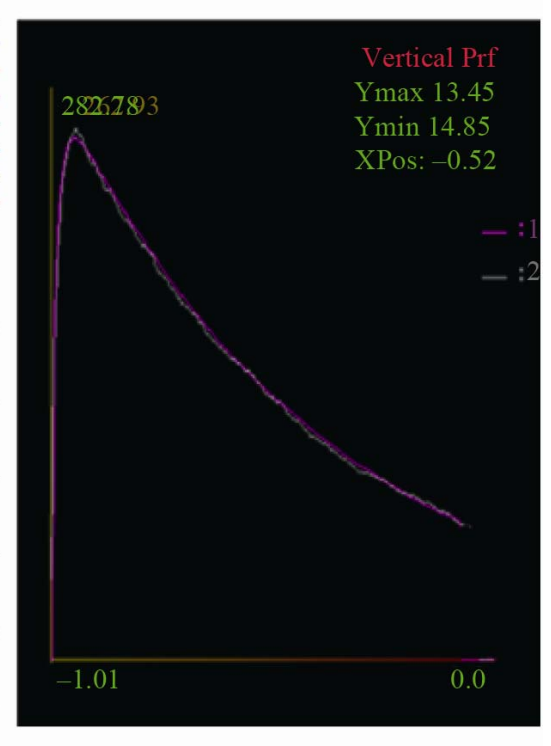

(b)

Figure 3. (a) $2 \times 2 \mathrm{~cm}$ MLC (3 $\times 3 \mathrm{~cm}$ jaw); (b) TP: pink, film: white. 




(a)

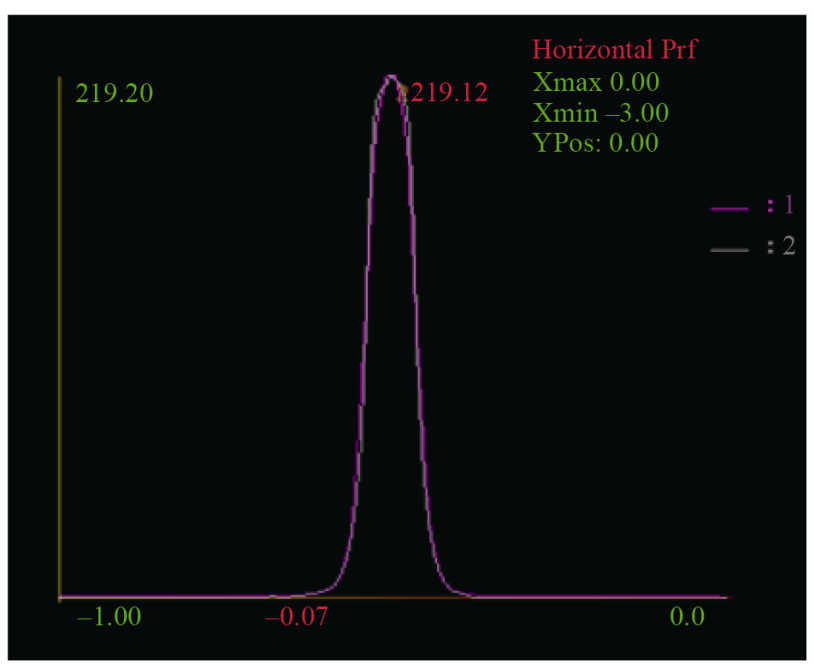

(b)

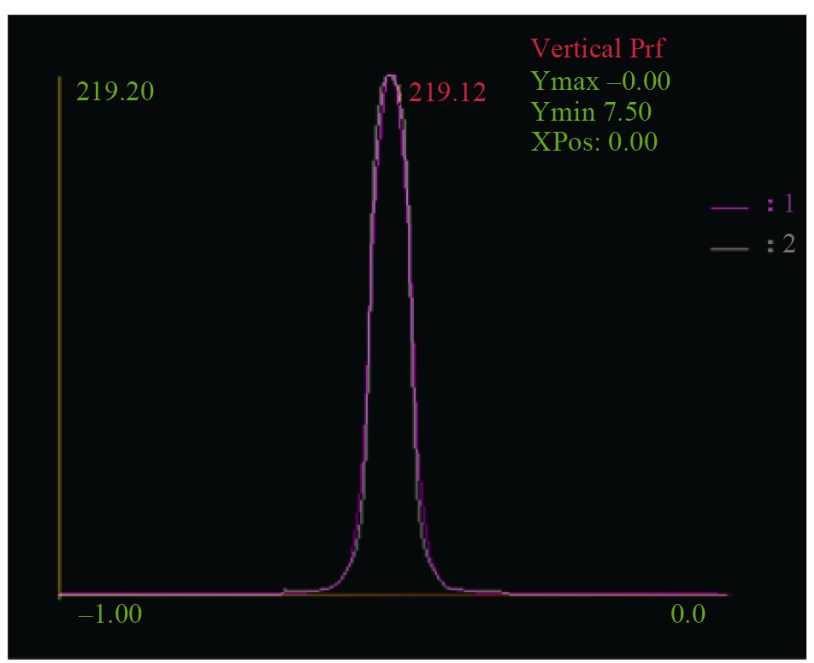

(c)

Figure 4. (a) $1 \times 1 \mathrm{~cm}$ isodoses overlay; passing rate $(2 \% / 2$ mm): $98.99 \%$; (b) $1 \times 1 \mathrm{~cm}$ crossplane profile; TP: pink, film: white; (c) $1 \times 1 \mathrm{~cm}$ inplane profile; TP: pink, film: white.

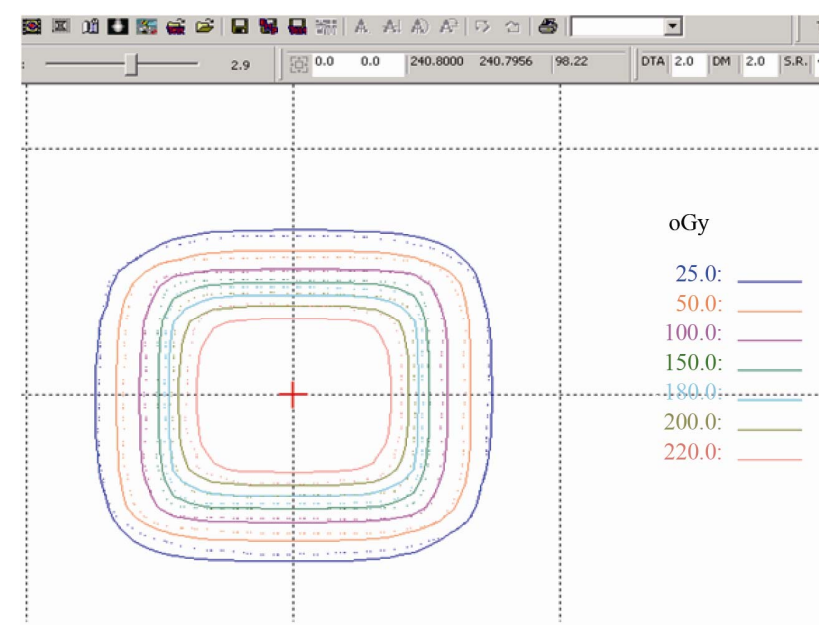

(a)

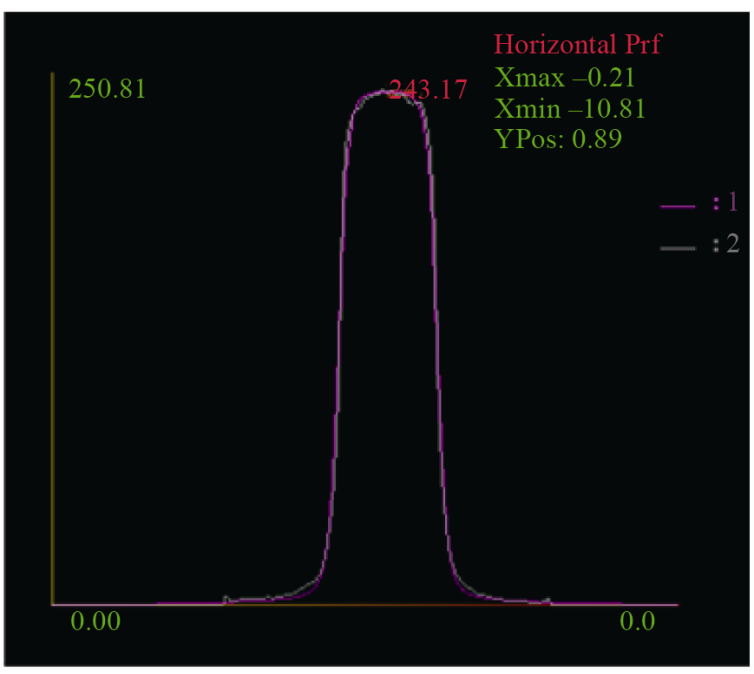

(b)

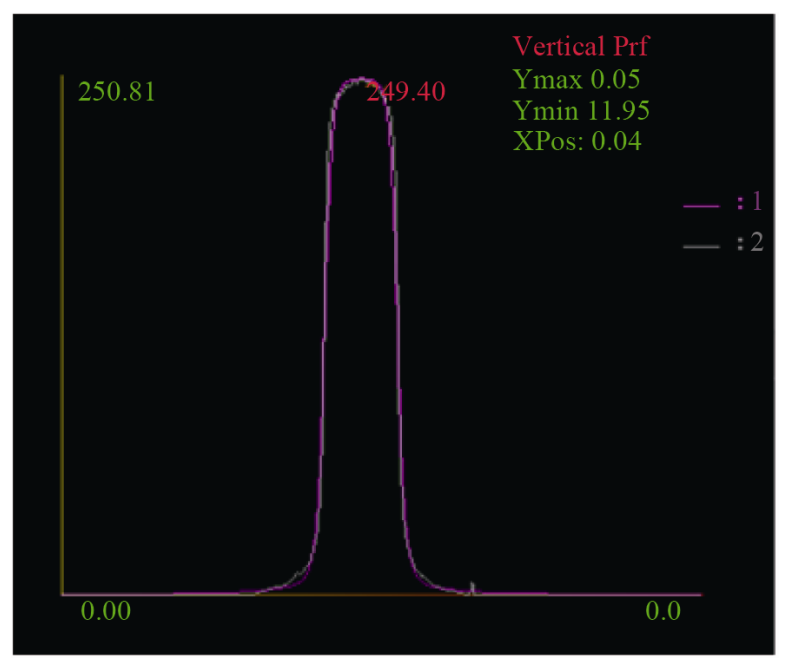

(c)

Figure 5. (a) $2 \times 2 \mathrm{~cm}$ isodoses overlay; passing rate $(2 \% / 2$ $\mathrm{mm}): 98.22 \%$; (b) $2 \times 2 \mathrm{~cm}$ crossplane profile; TP: pink, film: white; (c) $2 \times 2 \mathrm{~cm}$ inplane profile; TP: pink, film: white. 


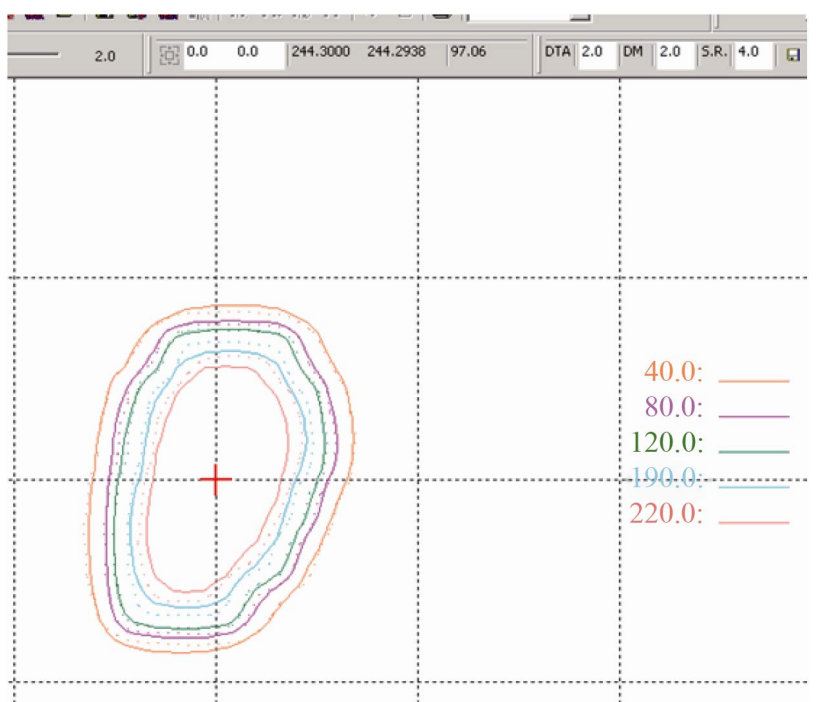

(a)

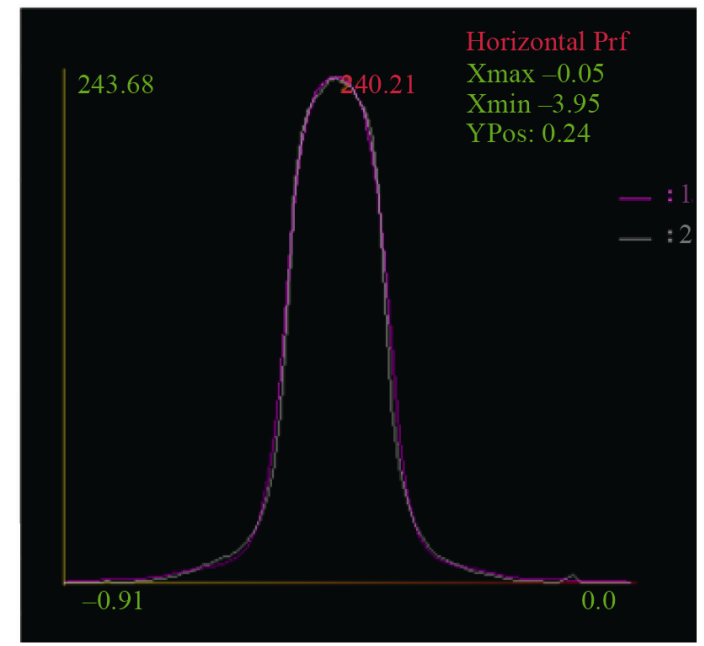

(b)

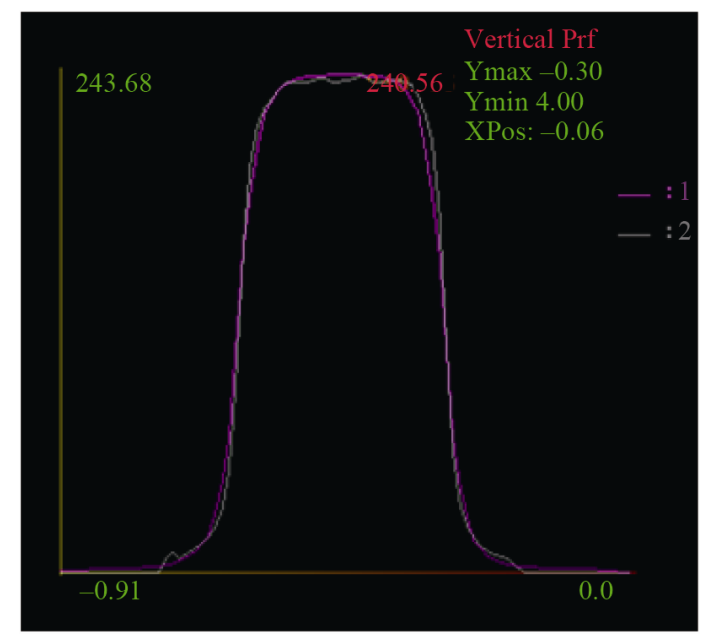

(c)

Figure 6. (a) SRS \#1 isodoses overlay; passing rate $(2 \% / 2$ mm): $97.06 \%$; (b) SRS \#1 crossplane profile; TP: pink, film: white; (c) SRS \#1 inplane profile; TP: pink, film: white.

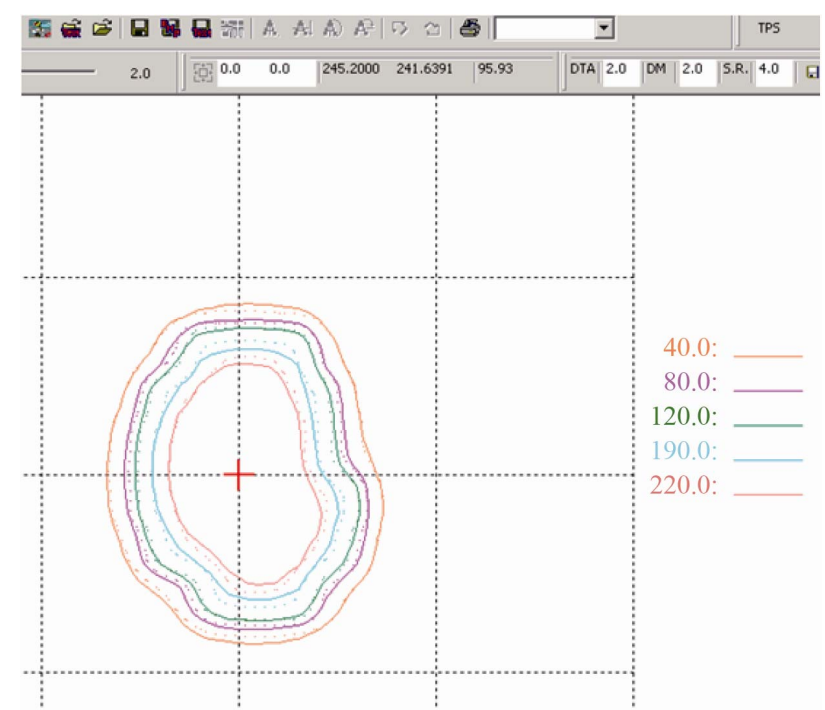

(a)

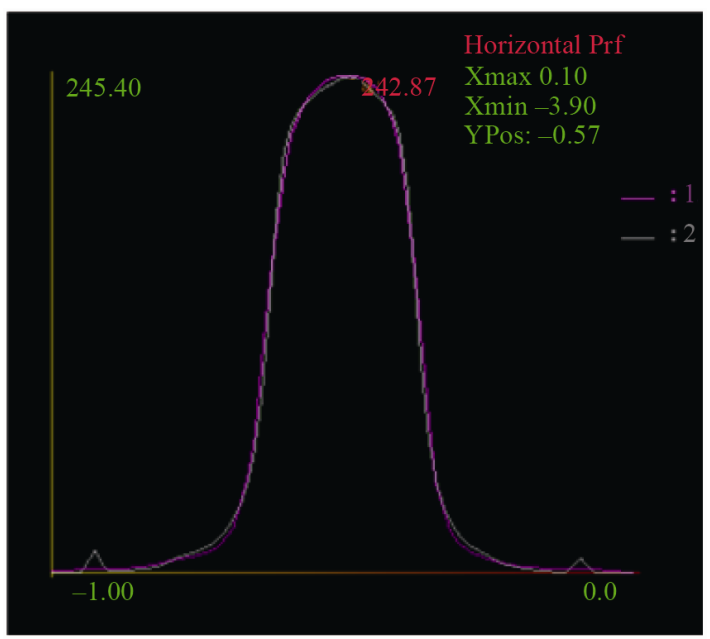

(b)

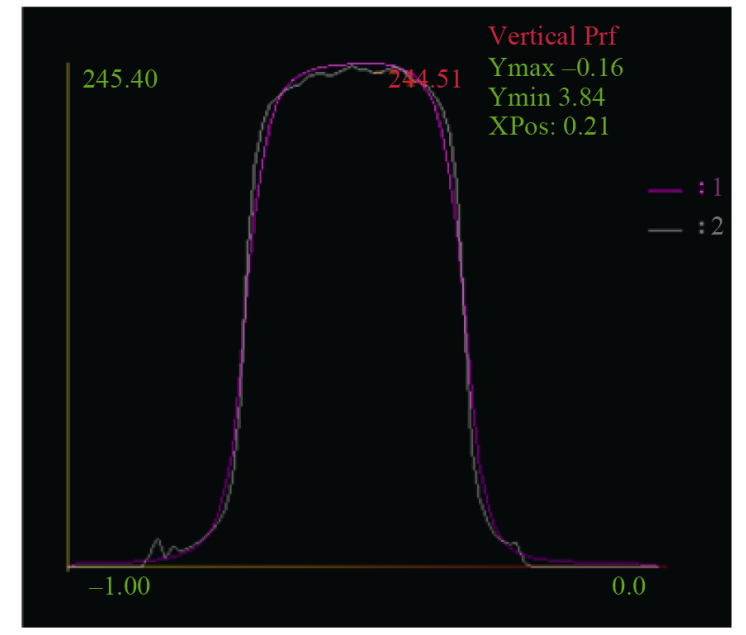

(c)

Figure 7. (a) SRS \#2 isodoses overlay; passing rate $(2 \% / 2$ mm): $95.93 \%$; (b) SRS \#2 crossplane profile; TP: pink, film: white; (c) SRS \#2 inplane profile; TP: pink, film: white. 
From our experience, it is important to assure the positioning of an EBT2 film edge at the surface of the polystyrene phantom to submillimeter accuracy. There can also be artifact of dosimetry near an edge of the film due to the mechanical insult caused by a cutting procedure [38]. Furthermore, EBT2 film could be used as a verification tool to compare the iPlan beam commissioning data provided by the manufacturer (BrainLab). After all, it is user's responsibility to confirm the accuracy of the commissioning data.

\section{Conclusions}

We have evaluated the measured dose distributions using EBT2 films for small fields in iPlan commissioning as well as patient-specific EBT2 film QA for iPlan SRS patients. The results are very satisfactory. The EBT2 films could be a useful dosimetry tools for small fields in SRS cases.

\section{REFERENCES}

[1] I. J. Das, G. X. Ding and A. Ahnesjo, "Small Fields: Nonequilibrium Radiation Dosimetry," Medical Physics, Vol. 35, No. 1, 2008, pp. 206-215. doi:10.1118/1.2815356

[2] A. E. Nahum, "Perturbation Effects in Dosimetry. i. Kilovoltage X-Rays and Electrons," Physics in Medicine and Biology, Vol. 41, No. 9, 1996, pp. 1531-1580. doi:10.1088/0031-9155/41/9/001

[3] M. B. Sharpe, D. A. Jaffray, J. J. Battista and P. Munro, "Extrafocal Raditation: A Unified Approach to the Prediction of Beam Penumbra and Output Factors for Megavolrage X-Ray Beams," Medical Physics, Vol. 22, No. 12, 1995, pp. 2066-2074. doi:10.1118/1.597648

[4] T. C. Zhu and B. E. Bjarngard, "The Head-Scatter Factor for Small Field Sizes," Medical Physics, Vol. 21, No. 1, 1994, pp. 65-68. doi:10.1118/1.597256

[5] T. C. Zhu and B. E. Bjarngard, "The Fraction of Photons Undergoing Head Scatter in X-Ray Beams," Physics in Medicine and Biology, Vol. 40, No. 6, 1995, pp. 11271134. doi:10.1088/0031-9155/40/6/011

[6] T. C. Zhu, B. E. Bjarngard and H. Shackford, "X-Ray Source and the Output Factor," Medical Physics, Vol. 22, No. 6, 1995, pp. 793-798. doi:10.1118/1.597588

[7] P. Francescon, S. Cora, C. Cavedon, P. Scalchi, S. Reccanello and F. Colombo, "Use of a New Type of Radiochromic Film, a New Parallel-Plate Micro-Chamber, Mosfets, and TLD 800 Microcubes in the Dosimetry of Small Beams," Medical Physics, Vol. 25, No. 4, 1998, pp. 503511. doi:10.1118/1.598227

[8] O. A. Sauer and J. Wilbert, "Measurement of Output Factors for Small Photon Beams," Medical Physics, Vol. 34, No. 6, 2007, pp. 1983-1988. doi:10.1118/1.2734383

[9] C. G. Soares, S. Trichter and S. Devic, "Radiochromic Film Dosimetry," In: D. W. O. Rogers and J. Cygler, Eds., Clinical Dosimetry for Radiotherapy, Medical Physics Publishing, Madison, 2009.
[10] S. Chiu-Tsao, D. Medich and J. Munro, "The Use of New GAFCHROMIC ${ }^{\circledR}$ EBT Film for ${ }^{125}$ I Seed Dosimetry in Solid Water ${ }^{\circledR}$ Phantom," Medical Physics, Vol. 35, No. 8, 2008, pp. 3787-3799. doi:10.1118/1.2955746

[11] M. Todorovic, M. Fischer, F. Cremers, E. Thom and Schmidt, "Evaluation of GafChromic EBT Prototype B for External Beam Dose Verification," Medical Physics, Vol. 33, No. 5, 2006, pp. 1321-1328. doi:10.1118/1.2188077

[12] F. C. Su, Y. Liu, S. Stathakis, C. Shi, C. Esquivel and N. Papanikolaou, "Dosimetry Characteristics of GAFCHROMIC $^{\circledR}$ EBT Film Responding to Therapeutic Electron Beams," Applied Radiation and Isotopes, Vol. 65, No. 10, 2007, pp. 1187-1192. doi:10.1016/j.apradiso.2007.05.005

[13] S. Chiu-Tsao, Y. Ho, R. Shanka, L. Wang and L. B. Harrison, "Energy Dependence of Response of New High Sensitivity Radiochromic Films for Megavoltage and Kilovoltage Radiation Energies," Medical Physics, Vol. 32, No. 11, 2005, pp. 3350-3354. doi:10.1118/1.2065467

[14] M. J. Butson, T, Cheung and P. K. N. Yu, "Weak Energy Dependence of EBT Gafchromic Tm Film Dose Response in the $50 \mathrm{KVp}-10 \mathrm{MVp}$ X-Ray Range," Applied Radiation and Isotopes, Vol. 64, No. 1, 2006, pp. 60-62. doi:10.1016/j.apradiso.2005.07.002

[15] J. G. H. Sutherland and D. W. O. Rogers, "Monte Carlo Calculated Absorbed-Dose Energy Dependence of EBT and EBT2 Film", Medical Physics, Vol. 37, No. 3, 2010, pp. 1110-1116. doi:10.1118/1.3301574

[16] S. Devic, J. Seuntjens, W. Abdel-Rahman, M. Evans, M. Olivares, E. B. Podgorsak, T. Vuong and C. G. Soares, "Skin Dose Measurement Using Radiochromic Film in Clinical Applications," Medical Physics, Vol. 33, No. 4, 2006, pp. 1116-1124. doi:10.1118/1.2179169

[17] M. J. Butson, T. Cheung and P. K. N. Yu, "Megavoltage $\mathrm{X}$-Ray Skin Dose Variation with an Angle Using Grid Carbon Fibre Couch Tops," Physics in Medicine and Biology, Vol. 52, No. 20, 2007, pp. 485-492.

[18] S. Price, M. Williams, M. Butson and P. Metcalfe, "Comparison of Skin Dose between Conventional Radiotherapy and IMRT," Australasian Physical and Engineering Sciences in Medicine, Vol. 29, No. 3, 2006, pp. 272-277. doi:10.1007/BF03178577

[19] H. Chung, H. Jin, J. F. Dempsey, C. Liu, J. Palta, T.-S. Suh and S. Kim, "Evaluation of Surface and Build-Up Region Dose for Intensity-Modulated Radiation Therapy in Head and Neck Cancer," Medical Physics, Vol. 32, No. 8, 2005, pp. 2682-2689. doi:10.1118/1.1992067

[20] S. Chiu-Tsao and M. F. Chan, "Evaluation of Two-Dimensional Bolus Effect of Immobilization/Support Devices on Skin Doses: A Radiochromic EBT Film Dosimetry Study in Phantom," Medical Physics, Vol. 37, No. 7, 2010, pp. 3611-3626. doi:10.1118/1.3439586

[21] L. J. van Battum, D. Hoffmans, H. Piersma and S. Heukelom, "Accurate Dosimetry with Gafchromic ${ }^{\mathrm{TM}}$ EBT Film of a 6 MV Photon Beam in Water: What Level Is Achievable?" Medical Physics, Vol. 35, No. 2, 2008, pp. 704-716. doi:10.1118/1.2828196

[22] C. Soares, "New Developments in Radiochromic Film Dosimetry," Radiation Protection Dosimetry, Vol. 120, 
No. 1-4, 2006, pp. 100-106. doi:10.1093/rpd/nci698

[23] S. Devic, N. Tomic, C. G. Soares and E. B. Podgorsak, "Optimizing the Dynamic Range Extension of a Radiochromic Film Dosimetry System," Medical Physics, Vol. 36, No. 2, 2009, pp. 429-437. doi:10.1118/1.3049597

[24] F. Andic, Y. Ors, R. Davutoglu, S. B. Cifci, E. B. Ispir and M. E. Erturk, "Evaluation of skin Dose Associated with Different Frequencies of Bolus Applications in PostMastectomy Three-Dimensional Conformal Radiotherapy," Journal of Experimental \& Clinical Cancer Research, Vol. 28, 2009, p. 41. doi:10.1186/1756-9966-28-41

[25] J. E. Lye, D. J. Butler and D. V. Webb, "Enhanced Epidermal Dose Caused by Localized Electron Contamination from Lead Cutouts Used in Kilovoltage Radiotherapy," Medical Physics, Vol. 37, No. 8, 2010, pp. 39353939. doi:10.1118/1.3458722

[26] M. Oldham, H. Sakhalkar, P. Guo and J. Adamovics, “An Investigation of the Accuracy of an IMRT Dose Distribution Using Two- and Three-Dimensional Dosimetry Techniques," Medical Physics, Vol. 35, No. 5, 2008, pp. 2072-2080. doi:10.1118/1.2899995

[27] D. Fontanarosa, L. C. Orlandini, I. Andriani and L. Bernardi, "Commissioning Varian Enhanced Dynamic Wedge in the Pinnacle Treatment Planning System Using Gafchromic $^{\text {TM }}$ EBT Film," Medical Physics, Vol. 36, No. 10, 2009, pp. 4504-4510. doi:10.1118/1.3223621

[28] J. Jr. Novotny, J. P. Bhatnagar, M. A. Quader, G. Bednarz, L. D. Lunsford and M. S. Huq, "Measurement of Relative Output Factors for the 8 and $4 \mathrm{~mm}$ Collimators of Leksell Gamma Knife Perfexion by Film Dosimetry," Medical Physics, Vol. 36, No. 5, 2009, pp. 1768-1774. doi: $10.1118 / 1.3113904$

[29] M. Fragoso, N. Wen, S. Kumar, D. Liu, S. Ryu, B. Movsas, M. Ajlouni and I. J. Chetty, “ Dosimetric Verification and Clinical Evaluation of a New Commercially Available Monte Carlo-Based Dose Algorithm for Application in Stereotactic Body Radiation Therapy (SBRT) Treatment Planning," Physics in Medicine and Biology, Vol. 55, No. 16, 2010, pp. 4445-4464. doi: $10.1088 / 0031-9155 / 55 / 16 / \mathrm{S} 02$

[30] O. A. Zeidan, S. A. L. Stephenson, S. L. Meeks, T. H. Wagner, T. R. Willoughby, P. A. Kupelian and K. M. Langen, "Characterization and Use of EBT Radiochromic
Film for IMRT Dose Verification," Medical Physics, Vol. 33, No. 6, 2006, pp. 4064-4072. doi:10.1118/1.2241047

[31] S. Chiu-Tsao and M. F. Chan, "Dose Response Characteristics of the New EBT-2 Film for Different Megavoltage Beams", Medical Physics, Vol. 36, No. 6, 2009, p. 2604. doi:10.1118/1.3181842

[32] M. J. Butson, P. K. N. Yu, T. Cheung and H. Alnawaf, "Energy Response of the New EBT2 Radiochromic Film to X-Ray Radiation," Radiation Measurements, Vol. 45, No. 7, 2010, pp. 836-839.

doi:10.1016/j.radmeas.2010.02.016

[33] B. Arjomandy, R. Tailor, A. Anand, N. Sahoo, M. Gillin, K. Prado and M. Vicic, "Energy Dependence and Dose Response of Gafchromic EBT2 Film over a Wide Range of Photon, Electron, and Proton Beam Energies," Medical Physics, Vol. 37, No. 5, 2010, pp. 1942-1947. doi:10.1118/1.3373523

[34] C. Andrés, A. del Castillo, R. Tortosa, D. Alonso and R. Barquero, "A Comprehensive Study of the Gafchromic EBT2 Radiochromic Film. A Comparison with EBT," Medical Physics, Vol. 37, No. 12, 2010, pp. 6271-6278. doi:10.1118/1.3512792

[35] T. Kairn, J. Kenny, S. B. Crowe, A. L. Fielding, R. D. Franich, P. N. Johnston, R. T. Knight, C. M. Langton, D. Schlect and J. V. Trapp, "Technical Note: Modeling a Complex Micro-Multileaf Collimator Using the Standard BEAMnrc Distribution," Medical Physics, Vol. 37, No. 4, 2010, pp. 1761-1767. doi:10.1118/1.3355873

[36] J. H. D. Wong, T. Knittel, S. Downes, M. L. F. Lerch, M. Petasecca, V. L. Perevertaylo, P. Metcalfe, M. Jackson and A. B. Rosenfeld, "The Use of a Silicon Strip Detector Dose Magnifying Glass in Stereotactic Radiotherapy QA and Dosimetry," Medical Physics, Vol. 38, No. 3, 2011, pp. 1226-1238. doi:10.1118/1.3549759

[37] S. Chiu-Tsao and M. F. Chan, "Photon Beam Dosimetry in the Superficial Buildup Region Using Radiochromic EBT Film Stack," Medical Physics, Vol. 36, No. 6, 2009, pp. 2074-2083. doi:10.1118/1.3125134

[38] P. K. N. Yu, M. J. Butson and T. Cheung, "Does Mechanical Pressure on Radiochromic Film Affect Optical Absorption and Dosimetry," Australasian Physical and Engineering Sciences in Medicine, Vol. 29, No. 3, 2006, p. 29. doi:10.1007/BF03178580 